\title{
DFT calculations in periodic boundary conditions of gas-phase acidities and of transition-metal anionic clusters: case study with carboxylate-stabilized ruthenium clusters
}

\author{
Roberto González Gómez ${ }^{1,2}$. Iker del Rosal ${ }^{2}$ - Karine Philippot ${ }^{1}$. \\ Romuald Poteau ${ }^{2(*)}$
}

the date of receipt and acceptance should be inserted later

\begin{abstract}
The first part of this study aims at evaluating the accuracy of DFT calculations of acids and their conjugate bases in periodic boundary conditions and plane wave basis sets. The resulting gas-phase acidities are compared to experimental data. Calculations done with the PBE functional are accurate, with a 4 kcal.mol ${ }^{-1}$ mean average deviation (MAD) with respect to experiments and a $\sim 1 \mathrm{kcal}^{\mathrm{mol}}{ }^{-1} \mathrm{MAD}$ with respects to non-periodic DFT calculations done in the aug-ccpvtz basis set with the same functional. The relative stability of $\mathrm{Ru}_{13}^{-}$isomers is also successfully compared to previous calculations done using local basis sets $(J$. Chem. Phys. (2015) 142, 024319). Finally, several carboxylic acids and their conjugate bases are adsorbed on two $\mathrm{Ru}_{13}$ clusters, showing a linear correlation between adsorption energies and experimental gas-phase acidities.
\end{abstract}

Keywords DFT · Ruthenium clusters · Gas-phase acidities

\section{Introduction}

In the past twenty five years, colloidal transition metal nanoparticles (TMNPs) have been strongly attracting the attention of the scientific community due to their unique properties located between those of bulk materials and molecules $[1,2]$. It is well-known that controlling the structure of nanomaterials can directly influence their physical and chemical properties, that can

${ }^{1}$ LCC-CNRS, Université de Toulouse, UPS, 205 route de Narbonne, BP 44099, F-31077-Toulouse Cedex 4, France . ${ }^{2}$ LPCNO (IRSAMC), Université de Toulouse; INSA, UPS, CNRS (UMR 5215); Institut National des Sciences Appliquées, 135 avenue de Rangueil, F-31077 Toulouse, France; (*) romuald.poteau@univtlse3.fr be of interest for applications in various fields ranging from biology, medicine, optoelectronics, catalysis, energy, etc[3]. The physical and chemical properties of TMNPs depend on their structural characteristics like their shape, size, defects, but also on the nature of capping ligands used to stabilize them and on the number of adsorbed species onto the metal surface. Among other transition metals, ruthenium is particularly appealing owing to its catalytic activity. Several RuNPs have been developed, both in solution and in supported conditions $[4,5]$. Despite this diversity, there is still a need to modify their surface in order to improve their properties and functionality. Although carboxylic acids are widely used as surfactants in colloidal synthesis, the direct adsorption of carboxylic acids onto ruthenium metal surfaces has received less attention. In solution, and according to their $p K_{\mathrm{a}}$, to the solvent and to $\mathrm{pH}$, carboxylic acids can dissociate into protons and carboxylates, although in water they are usually weak acids. It means that, while the usual picture is the dissociative adsorption of acids at the surface of TMNPs, carboxylates could directly adsorb on metal surfaces in appropriate experimental conditions and hence inject electrons in the electronic band structure of TMNPs.

Molecular physics techniques based on mass spectrometry offer a route to produce and characterize electronand atom-precise transition metal nanoclusters in gasphase. Bare $[6,7,8,9]$ or even hydrogen-protected[10] cluster anions can be generated by magnetron sputtering, selected by size and stored in a quadrupole ion trap in order to bring them to thermal equilibrium at low temperatures. Electron scattering measurements coupled to atomic modeling and to DFT calculations on anionic clusters allowed to evidence that different structural types exhibit unique experimentally distinguishable fingerprints. 
Being able to calculate the electronic and structural properties of charged species is thus of great interest, both in metal nanoparticles and metal clusters science. Among the first principles quantum mechanical softwares used in this field, VASP $[11,12]$ is a tool of choice, given its efficiency and its SCF convergence ability for zero-gap materials. Turbomole[13] has also been used to calculate the properties of anionic transition metal clusters at the DFT level of theory[10]. VASP, although specifically designed to describe bulk materials or surfaces by DFT methods with periodic boundary conditions (PBC), can also be applied to molecules, thanks to the supercell approach. It uses the projector augmented wave method, and a plane wave basis set. Yet, when PBC are applied to a supercell that contains a charged species, an electrostatic interaction occurs between the charge and its image in the neighbor supercells and contributes to the total energy of the system. This is a spurious, unwanted energy contribution, that must be canceled. It is for that purpose that all ioncontaining periodically replicated simulation cells have a neutralizing background, in effect adding a monopole correction to the total energy. This correction due to the background and the image charge interactions has been well documented $[14,15]$. In atomic units it is given by $E_{\text {Ewald }}=q^{2} \alpha / 2 L \epsilon_{0}$, where $\alpha$ is the Madelung constant, $q$ is the net charge of the system and $L$ is the length of the cubic unit cell. Such correction is available in VASP. However, to the best of our knowledge, no general study on the accuracy of this method for charged molecules or clusters has been achieved. It may be because VASP is generally not known as being the most appropriate program for molecular species. However, given that species grafted at the surface of TMNPs can modulate the properties of the metal core, assessing the ability of VASP to deliver accurate geometries and energies of charged metallic species, in addition to neutral compounds, is of utmost importance. The present study focuses on gas-phase acidities and geometries of selected organic compounds (section 3) and on the relative stability of anionic ruthenium isomers $\mathrm{Ru}_{13}^{-}$(section 4.1). After comparison to reference data, it will be shown that DFT calculations with PBC and plane wave basis sets performs also very well on these species. Given the very satisfactory results obtained, some clues are provided in section 4.2 about the adsorption energies of carboxylic acids and carboxylates at the surface of an icosahedral $\mathrm{Ru}_{13}$ core.

\section{Computational details}

\subsection{Gaussian basis set calculations}

All DFT calculations in local basis sets were performed with Gaussian09[16]. Geometries were fully optimized in gas phase without symmetry constraints, employing the Perdew-Burke-Ernzerhof (PBE) form[17, 18] of the generalized gradient approximation (GGA) and the aug-cc-pvtz correlation-consistent basis set[19, 20, 21]. Calculations of vibrational frequencies were systematically done in order to characterize the nature of stationary points. The vibrational analysis was also used to compute enthalpies in the framework of the harmonic approximation for the vibrational contribution (see for example ref. 22 for detailed thermodynamical equations).

\subsection{Plane-wave basis set calculations}

They were performed with the Vienna $a b$ initio simulation package, VASP[11, 12] within the framework of density functional theory. Projector augmented waves $(\mathrm{PAW})[23,24]$ were used, with a plane-wave kinetic energy cutoff of $550 \mathrm{eV}$. All the calculations used the PBE functional. The supercell used was $30 \times 30 \times 30 \AA$ large. $\Gamma$-centered[25] calculations were performed with a Gaussian smearing $(\sigma)$ of $0.01 \mathrm{eV}$, the energies being therefore extrapolated for $\sigma=0.00 \mathrm{eV}$. The atoms positions were optimized until the criterion of the residual forces on any direction being less than $0.01 \mathrm{eV} / \AA$ was met. A vibrational analysis has been systematically undertaken in order to characterize the nature of stationary points. A good accuracy of the vibrational frequencies calculation is obtained by setting up the threshold to stop the relaxation of the electronic degrees of freedom to $1 \times 10^{-8} \mathrm{eV}$, whilst the displacement of each ion to numerically calculate the Hessian Matrix is set up to $5 \times 10^{-3} \AA$. Enthalpies were computed with the home-made ThermoWithVASP tool, following the same methodology as the one used in Gaussian09. The main approximation relies on non-interacting molecules and therefore applies only to ideal gases. It is however often also applied to condensed matter. In short, enthalpies are calculated by summing $R T$ (i.e. $P V$ ) and a thermal correction to DFT energies, $E_{\text {therm }}$. $E_{\text {therm }}$ is deduced from a straightforward application of statistical thermodynamics, i.e. by calculating the molecular partition function $q(V, T)[22]$. If one assumes that there is a unique molecular conformation under temperature $T$ and a negligible coupling between the translational and rotational motions and the electronic states occupation and the vibrational motion, $q$ can be separated into a 
product of translational, rotational, electronic and vibrational partition functions. As a consequence, $E_{\text {therm }}$ is calculated as $E_{\text {therm }}=E_{\text {vib }}+E_{\text {rot }}+E_{\text {trans }}+E_{\text {elec }}$, where each contribution $E_{\mathrm{X}}$ to the thermal correction ( $\mathrm{X}=\mathrm{vib}$, rot, trans, elec) is evaluated from the partition function $q_{\mathrm{X}}$ :

$E_{\mathrm{X}}(T)=R T^{2}\left(\frac{\partial \ln \left(q_{\mathrm{X}}\right)}{\partial T}\right)_{V}$

Tests were made to check that the same data provide the same thermal corrections to DFT energies than Gaussian09.

The Density of States (DOS), d-band centers[26, 27] and Crystal Orbital Hamilton Population (COHP)[28] of ruthenium clusters were calculated after projection of the PAW wavefunction in a minimal basis set. It was achieved with the Lobster software, using the pbeVASPfit basis set[29, 30, 31]. Integrated pCOHP indexes between two atoms $\mathrm{A}$ and $\mathrm{B}$ were also calculated as:

$\operatorname{IpCOHP}_{\mathrm{AB}}=\sum_{\mu \in \mathrm{A}} \sum_{\nu \in \mathrm{B}} \int_{E_{\mathrm{min}}}^{E_{\mathrm{F}}} \operatorname{pCOHP}_{\mu \nu}(\varepsilon) d \varepsilon$

where $\mu$ and $\nu$ run over all AOs belonging to atoms $\mathrm{A}$ and $\mathrm{B}$.

It provides a qualitative information of the average bond strength in covalent systems. The charge spilling, a criterion that assesses the quality of the projection, was systematically lower than $1.0 \%$. Atomic charges can be calculated by integrating the pDOS up to the Fermi energy. It is nothing else than a Mulliken population analysis (MPA) done with an orthogonal basis set. It is called pMPA in the paper. Some examples of pCOHP, IpCOHP, and pDOS electronic structure and bonding analysis of bare and ligand-protected RuNPs can be found in Refs. 32 and 33, whereas assessment of pMPA analysis is done in Ref. 34 .

\section{Gas-phase acidities}

The gas phase acidity is defined as the positive enthalpy change $\left(\Delta_{a} H^{\circ}\right)$ associated with the deprotonation of a molecule $\mathrm{AH}$ to form $\mathrm{A}^{-}$and $\mathrm{H}^{+}$ions: $\mathrm{AH}(\mathrm{g}) \longrightarrow$ $\mathrm{A}^{-}(\mathrm{g})+\mathrm{H}^{+}(\mathrm{g})$. As a reminder, the gas phase proton affinity (PA) is defined as the negative enthalpy change associated with the protonation of a molecule $\mathrm{B}$ to form the $\mathrm{BH}^{+}$ion: $\mathrm{B}(\mathrm{g})+\mathrm{H}^{+}(\mathrm{g}) \longrightarrow \mathrm{BH}^{+}(\mathrm{g})$. In the following, $\Delta_{a} H_{298}^{\circ}$ is calculated for the ten compounds shown in Fig. 1 as well as for water and the hydronium ion. Interestingly, and according to G4(MP2)$6 \mathrm{X}$ calculations[35], the conjugate base of molecule $\mathbf{9}$, the ortho-diethynylbenzene dianion (ortho- $\mathrm{DEB}^{2-}$ ), is to date the synthesized ion with the highest calculated

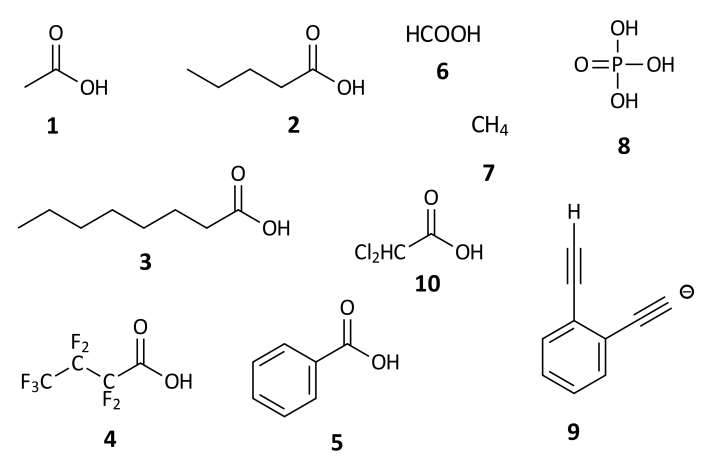

Fig. 1 Acids whose gas-phase acidity has been calculated in this study.

proton affinity $\left(\Delta_{a} H_{298}^{\circ}: 440.6 \mathrm{kcal} \cdot \mathrm{mol}^{-1}\right)$. With an experimental $\Delta_{a} H_{298}^{\circ}=321.9 \mathrm{kcal}^{\circ} \mathrm{mol}^{-1}[36]$, the heptafluorobutanoic acid, 4 , stands on the other side of the acidity range considered in this work. The convergence of the periodic-boundary PBE calculation in plane-wave basis set is first going to be evaluated for ethanoic acid as a function of the size of the supercell. $\Delta_{a} H_{298}^{\circ}$ acidities calculated with VASP will then be compared with reference data[37, 36, 38, 39, 40, 41], as well as with non-periodic PBE calculations done with Gaussian 09, with the aug-cc-pvtz basis set, which can be considered as close enough to the basis set limit. Some characteristic geometrical parameters obtained with the two theoretical methods will then be compared.

3.1 Convergence of the energy as a function of the size of the supercell

The energy of ethanoate with the correction involved by a neutralizing background is reported in Fig. 2 as a function of the size of the supercell, $L$, with $L$ in the range $8 \AA$ to $45 \AA$. Unsurprisingly, the correction to the energy is ill-defined at $8 \AA$. The corrected energy starts to smoothly converge at $15 \AA$. The corrected energy difference between the $30 \AA$ and $45 \AA$ supercell is $7 \times 10^{-3} \mathrm{eV}\left(\sim 0.2 \mathrm{kcal} . \mathrm{mol}^{-1}\right)$ only. From now on, all calculations will be performed in a $L=30 \AA$ box and energies will be given in kcal.mol ${ }^{-1}$.

\subsection{Comparison of $\Delta_{a} H_{298}$ energies with reference} data

All energies $\Delta E$ and acidities $\Delta_{a} H_{298}^{\circ}\left(\Delta_{a} H_{298}^{\circ}=\Delta E+\right.$ $\left.\Delta(P V)+\Delta E_{\text {therm }}\right)$ calculated with VASP or Gaussian are reported in Figure 3 and tabulated in Table 1, in comparison to experimental reference data. No experimental gas-phase acidity has been found in the literature for octanoic acid, and only a non-periodic PBE 


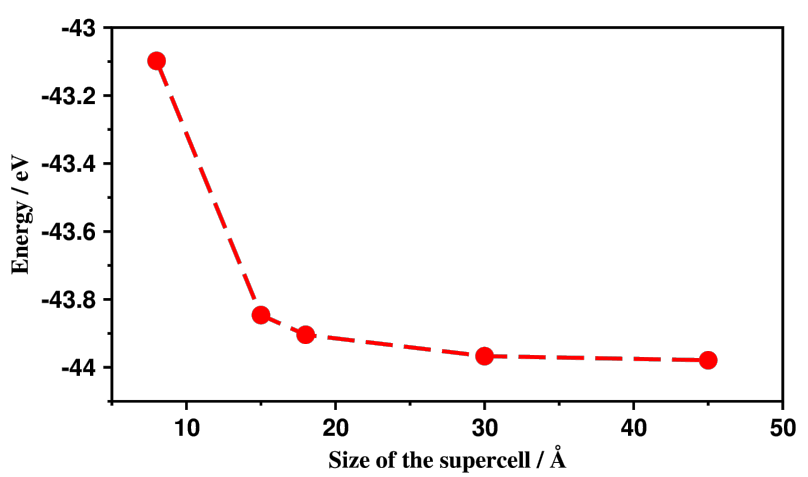

Fig. 2 Evolution of the energy of ethanoate as a function of the size of the supercell.

against periodic PBE comparaison has been made in table 1 .

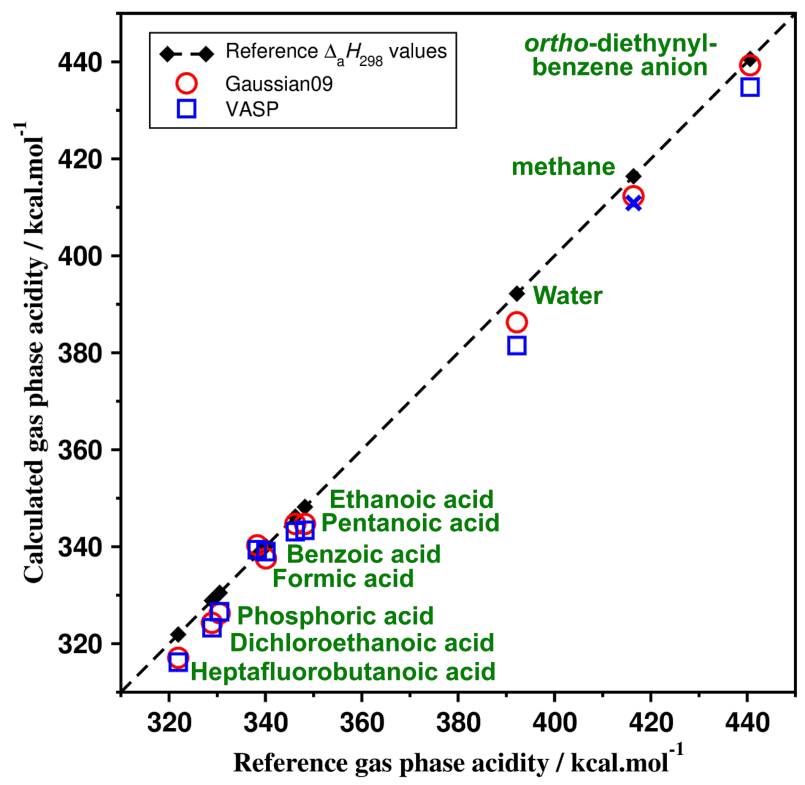

Fig. 3 Comparison of gas-phase acidities $\Delta_{a} H_{298}$ calculated in this work with reference data (formic acid, ethanoic acid, dischloroethanoic acid, pentanoic acid, heptafluorobutanoic acid: ref. 36; ortho-diethynylbenzene anion: ref. 35; methane: ref. 41; phosphoric acid: ref. 38). Dashed line corresponds to slope = 1 , i.e. to a perfect agreement between the theoretical calculations from this work and reference data. With $\Delta_{a} H_{298}^{\circ}=165.2$ kcal.mol ${ }^{-1}, \mathrm{H}_{3} \mathrm{O}^{+}$does not appear on this scale (see values in Table 1 ). Blue cross: only $\Delta E$ has been calculated with VASP (see text for details).

At first sight there is an overall very good agreement between the two theoretical approaches and only small deviations with respect to reference values. The two exceptions are hydroxide and the methyl anion. As for $\mathrm{HO}^{-}$, we have checked that using in VASP harder potentials for oxygen and setting up higher energy cutoffs do not improve the agreement between theory and experiments (VASP vs. experiments: $10.6 \mathrm{kcal}_{\mathrm{mol}} \mathrm{m}^{-1}$ ) or even with the aug-cc-pvtz calculations (VASP vs. G09: $4.7 \mathrm{kcal}^{\mathrm{mol}}{ }^{-1}$ ). Mind that even with non-periodic DFT methods, the calculation of $\Delta_{a} H_{298}$ for $\mathrm{HO}^{-}$is very sensitive to the basis set. The methyl anion was also uneasy to calculate with VASP. Given convergence issues in the numerical calculation of frequencies, it is the deprotonation energy, $\Delta E$, that is reported in Figure 3 for the methyl anion (blue cross). Owing to the singularity of $\mathrm{HO}^{-}$and $\mathrm{CH}^{-}$in the test set, they have not been considered in the calculation of mean average deviations (MAD) given hereafter. Deprotonation energies, $\Delta E$, calculated with VASP and G09 agree very well, with a $\mathrm{MAD}=0.9 \mathrm{kcal} . \mathrm{mol}^{-1}$. Although the numerical differentiation used in VASP to calculate vibrations is somewhat less accurate than the analytic calculations of second derivatives in G09, $\Delta_{a} H_{298}$ values calculated with VASP are very close to the non-periodic DFT values, with a MAD that slightly increases to $1.2 \mathrm{kcal}_{\mathrm{mol}}{ }^{-1}$. It also confirms the correctness of the ThermoWithVASP utility.

\subsection{Geometries}

All geometries and selected geometrical parameters are reported in Figure 4. In the following, discussions on geometry parameters will systematically be made by comparing G09 results with VASP. Although $\mathrm{HO}^{-}$appeared as a singularity in the calculation of the acidity of water, with a $\sim 5 \mathrm{kcal}^{\mathrm{mol}}{ }^{-1}$ energy difference between the two methods, as for geometries the agreement is excellent $(0.975 \AA$ vs. $0.976 \AA)$. The VASP geometry of the second singular anion, $\mathrm{CH}_{3}^{-}$, is not very accurate. It is in particular significantly less pyramidal than found with G09 (pyramidalization angle: $119.4^{\circ}$ vs. $135.4^{\circ}$ ). Apart from the methyl anion, G09 and VASP yield almost the same geometries for all remaining species considered in this work. The bond lengths in the hydronium ion are found to be the same within $1 / 1000 \AA(0.988$ $\AA$ ), whereas the dihedral angle $\omega$ is slightly higher with VASP $\left(125.6^{\circ}\right.$ vs. $\left.128.9^{\circ}\right)$. Regarding carboxylic acids (1-6, 10), both methods find the same expected trends, usually within a few thousandth of $\AA$ : HOOC-CR ${ }_{2}$ bond lengths increase upon deprotonation (for example: pentanoic acid $1.510 \AA$ vs. $1.510 \AA$ and pentanoate 1.574 $\AA$ vs. $1.572 \AA$, benzoic acid $1.487 \AA$ vs. $1.486 \AA$ and benzoate $1.554 \AA$ vs. $1.552 \AA)$; HO-C $(\mathrm{O})(\mathrm{R})$ bond lengths decrease upon deprotonation (for example: pentanoic acid $1.369 \AA$ vs. $1.370 \AA$ and pentanoate $1.264 \AA$ vs. $1.267 \AA$, benzoic acid $1.368 \AA$ vs. $1.370 \AA$ and benzoate $1.263 \AA$ vs. $1.267 \AA$ ); the two C-O bond lengths are not identical in some carboxylates owing to the strong polarization by electronegative atoms (for example, heptafluorobutanoic acid $1.252 \AA$ and $1.246 \AA$ vs. 1.255 
Table 1 Energy differences $\Delta E$ and gas-phase acidities $\Delta_{a} H_{298}^{\circ}$, in kcal.mol ${ }^{-1}$ of the reaction $\mathrm{AH}(\mathrm{g}) \longrightarrow \mathrm{A}^{-}(\mathrm{g})+\mathrm{H}^{+}(\mathrm{g})(\mathrm{or}$ $\mathrm{AH}^{+}(\mathrm{g}) \longrightarrow \mathrm{A}(\mathrm{g})+\mathrm{H}^{+}(\mathrm{g})$ for $\left.\mathrm{H}_{3} \mathrm{O}^{+}\right)$. The MAD is between calculations and reference data. ${ }^{\dagger}$ The gas-phase acidities of water and methane are not taken into account in the calculation of the MAD.

a: Pulsed electron high pressure mass spectrometer experiments, extrapolated from $T=600 \mathrm{~K}$, ref. 36

b: Energy-resolved, competitive threshold collision-induced dissociation experiments, $T=298 \mathrm{~K}$, ref. 40

c: Negative ion photoelectron velocity-map imaging experiments, $T=298 \mathrm{~K}$, ref. 41

d: Ion-molecule bracketing experiments, ref. 38

e: G4(MP2)-6X calculations, $T=298 \mathrm{~K}$, ref. 35

f: theoretically-corrected ionization threshold measurements, $T=298 \mathrm{~K}$, ref 39

g: JANAF tables, ref 37

See also the NIST database, 42.

\begin{tabular}{|c|c|c|c|c|c|}
\hline \multirow{2}{*}{ compound } & \multicolumn{2}{|c|}{ Gaussian09 } & \multicolumn{2}{|c|}{ VASP } & \multirow{2}{*}{ ref. $\Delta_{a} H^{\circ}$} \\
\hline & $\Delta E$ & $\Delta_{a} H_{298}^{\circ}$ & $\Delta E$ & $\Delta_{a} H_{298}^{\circ}$ & \\
\hline 1 & 351.7 & 344.7 & 351.7 & 343.2 & $348.5^{\mathrm{a}}, 348.2^{\mathrm{b}}$ \\
\hline 2 & 350.7 & 343.5 & 350.9 & 342.1 & $346.2^{\mathrm{a}}$ \\
\hline 3 & 350.4 & 343.1 & 351.0 & 341.1 & - \\
\hline 4 & 323.9 & 317.1 & 323.1 & 314.5 & $321.9^{\mathrm{a}}$ \\
\hline 5 & 344.7 & 337.6 & 345.1 & 336.8 & $340.1^{\mathrm{a}}$ \\
\hline 6 & 347.4 & 340.3 & 347.1 & 338.9 & $345.3^{\mathrm{a}}$ \\
\hline 7 & 420.6 & 412.3 & 410.9 & - & $416.4^{\mathrm{c}}$ \\
\hline 8 & 332.4 & 326.3 & 332.6 & 323.8 & $330.5^{\mathrm{d}}$ \\
\hline 9 & 445.4 & 439.3 & 440.3 & 434.8 & $440.6^{\mathrm{e}}$ \\
\hline 10 & 330.1 & 323.3 & 331.1 & 324.3 & $328.9^{\mathrm{a}}$ \\
\hline $\mathrm{H}_{3} \mathrm{O}^{+}$ & 170.6 & 164.0 & 171.2 & 164.5 & $165.2^{\mathrm{f}}$ \\
\hline $\mathrm{H}_{2} \mathrm{O}$ & 392.8 & 386.3 & 388.6 & 381.5 & $392.2^{\mathrm{g}}$ \\
\hline $\mathrm{MAD}^{\dagger}$ & & 3.4 & & 4.0 & 0.0 \\
\hline
\end{tabular}

$\AA$ and $1.249 \AA$ ). A careful reading of Figure 4 does not reveal any major discrepancy between the two methods. Let us now turn to phosphoric acid (8), a weak acid as well, and its conjugate base, dihydrogen phosphate. Bond lengths are similar within one hundredth of $\AA$ and angles differ by at most $0.2^{\circ}$. The CC bond lengths in the ortho- $\mathrm{DEBH}^{-}$compound (10) and its the conjugate base are found to be very similar with both methods. This result, together with the accuracy already observed in the calculation of the acidity with VASP, involves that the neutralizing background and the correction to the energy is also valid for the ortho$\mathrm{DEB}^{2-}$ dianionic species.

\section{Relative stability of neutral and anionic $\mathrm{Ru}_{13}$ isomers}

4.1 Convergence of the energy as a function of the size of the supercell

The $\left(\mathrm{CHCl}_{2} \mathrm{COO}^{*} \mathrm{Ru}_{13}-I_{\mathrm{h}}\right)^{-}$cluster has been used as a test case. The total energy is reported in Figure 6 as a function of $L$. With a $2.5 \mathrm{kcal}^{\mathrm{mol}}{ }^{-1}$ energy difference only between the $30 \AA$ and $45 \AA$ supercells, $30 \AA$ can be considered as good cost/accuracy compromise.

\subsection{Bare clusters}

The structures of small, bare ruthenium anionic clusters, $\mathrm{Ru}_{n}^{-}(n=8-20)$, were investigated a couple of years ago[43] using a combination of trapped ion electron diffraction (TIED) and density functional theory computations performed with the non-periodic Turbomole software[13]. The present study more particularly focuses on $\mathrm{Ru}_{13}^{-}$. According to electron diffraction measurements, its most stable isomer forms a $C_{2 \mathrm{v}}$ defective double layered hexagonal structure (see Figure 5), which was found to be the most stable by TPSS[44]/def2$\mathrm{SVP}$ [45] calculations. The second isomer $\left(C_{\mathrm{s}}-1\right)$ is based on the simple cubic motif. Although is was found to be isoenergetic to the $C_{2 \mathrm{v}}$ isomer, only the latter fit the TIED experimental data. A $C_{\mathrm{s}}-2$ isomer, a variant to $C_{\mathrm{s}}-1$, was also found, lying $0.1 \mathrm{eV}$ above the two degenerate isomers. The icosahedron $\left(I_{\mathrm{h}}\right)$ was not considered, probably owing to its high energy w.r.t. the low-lying isomers. Table 2 shows the relative energies $\Delta E$ (in kcal.mol ${ }^{-1}$ ) as well as the magnetic moments $\mu$ found in Ref. 43 at the TPSS/def2-SVP level of calculation. $\Delta E$ and $\mu$ calculated with VASP, using the same meta-GGA TPSS functional and a $500 \mathrm{eV}$ cut-off, are also reported in Table 2. Magnetic moments are found to be the same and $\Delta E$ differ at most by $2.9 \mathrm{kcal} . \mathrm{mol}^{-1}$. This difference could either be related to the basis set, to the size $L$ of the supercell or to the energy correction of the neutralizing background. The agreement is anyway very good and it shows that accurate DFT cal- 


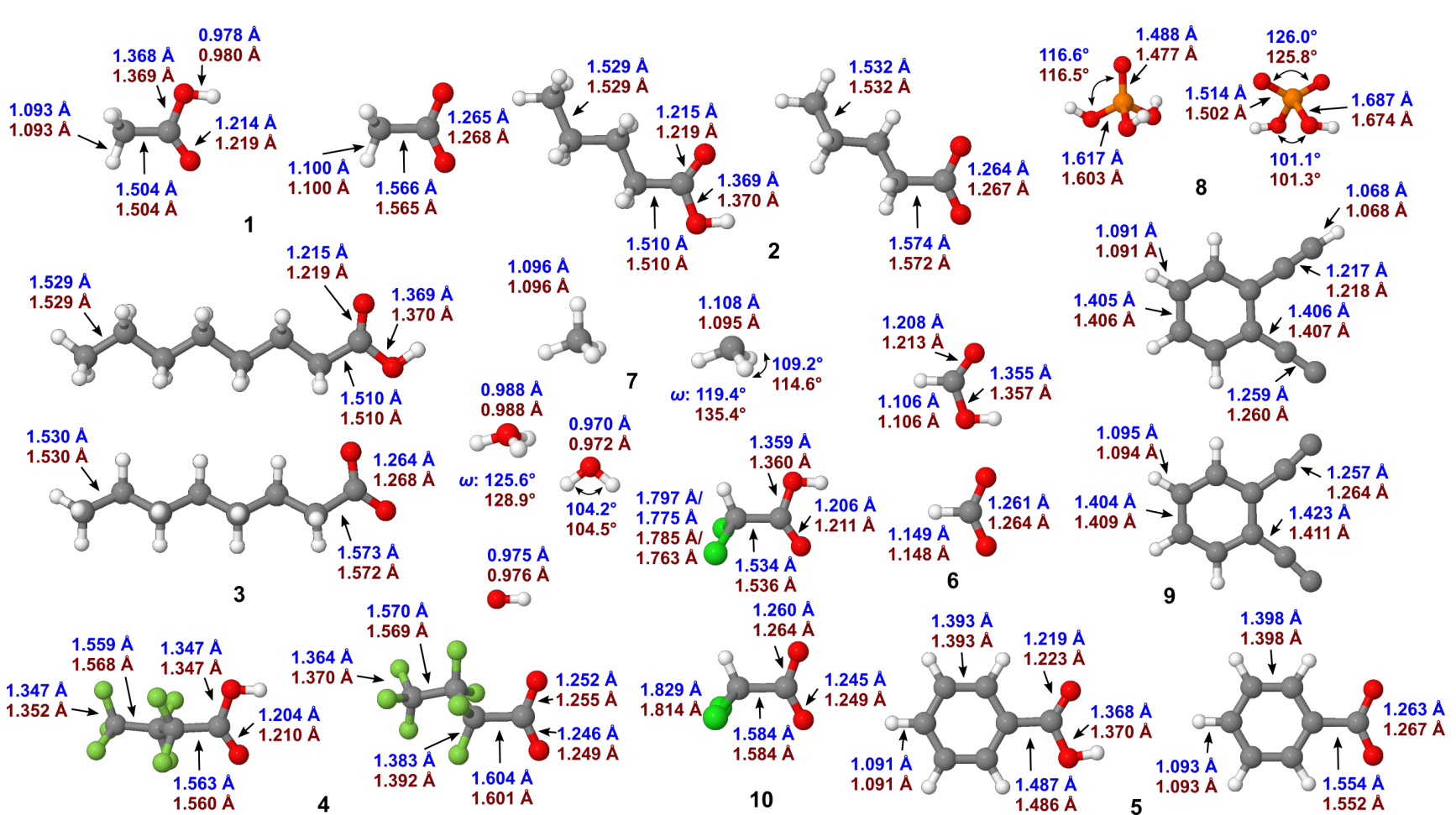

Fig. 4 Comparison of some geometrical parameters obtained with G09 (1st line, blue) and VASP (2nd line, burgundy).

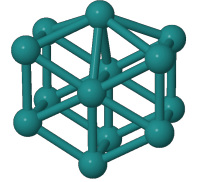

$\mathrm{C}_{2 \mathrm{v}}$

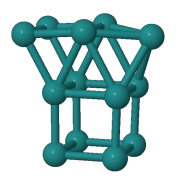

$\mathrm{C}_{\mathrm{s}}-1$

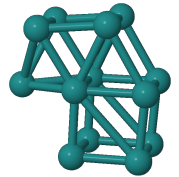

$\mathrm{C}_{\mathrm{s}}-\mathbf{2}$

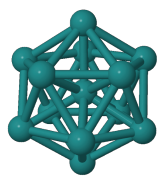

$\mathbf{I}_{\mathrm{h}}$

Fig. 5 Lowest energy isomers of $\mathrm{Ru}_{13}^{-}$clusters considered in this work (after ref. 43).

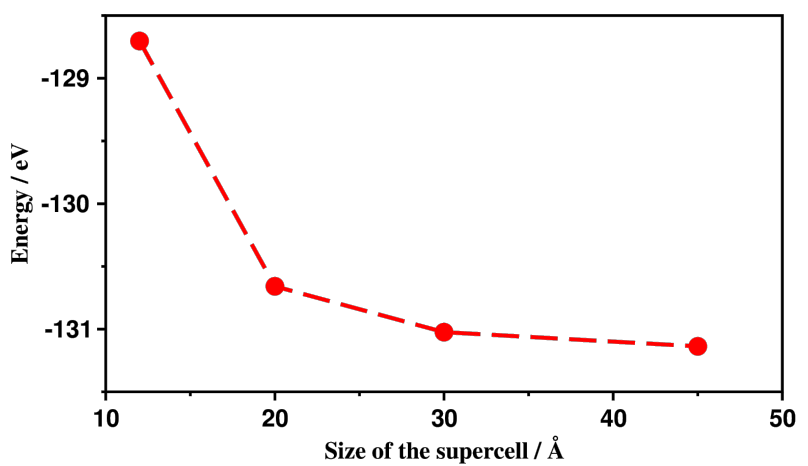

Fig. 6 Evolution of energies of dichloroethanoate adsorbed on $\mathrm{Ru}_{13}-I_{\mathrm{h}}$ as a function of the size of the supercell.

culations with PBC can be performed on anionic TM clusters. Incidentally, the $I_{h}$ cluster lies $c a .52 \mathrm{kcal} \mathrm{mol}^{-1}$ above the $C_{2 \mathrm{v}}$ isomer.

These last years, we have explored the surface and catalytic properties of RuNPs using the PBE functional[46, a $47,32,33,48]$ with PBC, which, at least to us, makes

Table 2 Symmetries, relative energies (in kcal. $\mathrm{mol}^{-1}$ ), and magnetic moments $\mathrm{f}\left(\right.$ in $\left.\mu_{\mathrm{B}}\right)$ or the lowest isomers of $\mathrm{Ru}_{13}^{-}$. a: def2-SVP gaussian basis set, ref. 43 .

\begin{tabular}{|c|c|c|c|c|c|c|}
\hline \multirow[b]{2}{*}{ symmetry } & \multicolumn{4}{|c|}{ TPSS } & \multicolumn{2}{|c|}{ PBE } \\
\hline & $\Delta E^{\mathrm{a}}$ & $\mu^{\mathrm{a}}$ & $\Delta E$ & $\mu$ & $\Delta E$ & $\mu$ \\
\hline$C_{2 \mathrm{v}}$ & 0.0 & 7 & 0.0 & 7 & 0.0 & 7 \\
\hline$C_{\mathrm{s}^{-1}}$ & 0.0 & 7 & 1.5 & 7 & -4.7 & 7 \\
\hline$C_{\mathrm{s}}-2$ & 2.3 & 7 & 5.2 & 7 & 1.0 & 7 \\
\hline$I_{\mathrm{h}}$ & - & - & 51.9 & 1 & 67.4 & 1 \\
\hline
\end{tabular}

the evaluation of this functional on charged species important for the sake of comparison with a large amount of data. The present results could also be compared to reference calculations of adsorption properties on $\mathrm{Ru}$ surfaces.[49] $\Delta E$ and $\mu$ calculated at this level of theory are also reported in Table 2 . The relative stability of the $C_{2 \mathrm{v}}$ and $C_{\mathrm{s}}-1$ isomers is switched between TPSS and PBE, the $C_{\mathrm{s}^{-1}}$ isomer being now the most stable isomer by $4.7 \mathrm{kcal}^{\mathrm{mol}}{ }^{-1}$. PBE is however accurate enough for the identification of the most interesting candidates that can account for the experimental TIED data. By the way, and regarding the use of TPSS for describing anionic ruthenium clusters, it has been claimed to "provide a relative energy accuracy to within several tenths of an electron volt"[9]. Besides, ZPE and thermal corrections could give a different ordering - this is however beyond the scope of the present study. Finally, TPSS , and PBE agree that the bare $I_{\mathrm{h}}$ isomer is high in energy, with a magnetic moment equal to $1 \mu_{\mathrm{B}}$. 
The pCOHP and pDOS profiles of the optimized neutral and anionic $C_{2 \mathrm{v}}$ clusters are reported in Figure 7. The Fermi energy is higher by $c a .2 \mathrm{eV}$ in $\mathrm{Ru}_{13}^{-}$, which a $d$-band center slightly closer than in $\mathrm{Ru}_{13}$. In average, the additional electron is equally shared by the $4 \mathrm{~d}$ and $5 \mathrm{~s}$ AOs. Whilst pDOS profiles of both compounds look alike below the Fermi energy, pCOHP profiles differ around it. Moreover, the IpCOHP index, found to be $1.04 \mathrm{eV}$ per $\mathrm{Ru}-\mathrm{Ru}$ bond in $\mathrm{Ru}_{13}$ is lowered to $0.98 \mathrm{eV}$ in $\mathrm{Ru}_{13}^{-}$, in agreement with the anti-bonding character of the available empty MOs in $\mathrm{Ru}_{13}$.

4.3 $\mathrm{Ru}_{13}-\mathrm{I}_{\mathrm{h}}$ and $\mathrm{Ru}_{13}-\mathrm{C}_{\mathrm{s}}-1$ clusters stabilized by carboxylic acids or carboxylates

Although the $I_{\mathrm{h}}$ isomer is much higher in energy than the low-lying $C_{2 \mathrm{v}}$ and $C_{\mathrm{s}}$ isomers, it is an interesting cluster to graft ligands on, owing to a core/surface structure that preludes larger clusters and NPs. The $C_{\mathrm{s}}-1$ isomer, although not observed experimentally in TIED experiments, is also an interesting test case owing to its simple cubic motif-based structure. We are now going to examine two adsorption mechanisms, namely the dissociative adsorption energy of gas-phase carboxylic acids on the metal surface (eq. 3), and the direct adsorption of gas-phase carboxylates (eq. 4).

$$
\begin{aligned}
& \mathrm{Ru}_{13}+\mathrm{CH}_{3} \mathrm{COOH} \rightarrow\left(\mathrm{Ru}_{13}\right)\left(\mathrm{CH}_{3} \mathrm{COO}\right)(\mathrm{H}) \\
& \mathrm{Ru}_{13}+\mathrm{CH}_{3} \mathrm{COO}^{-} \rightarrow\left[\left(\mathrm{Ru}_{13}\right)\left(\mathrm{CH}_{3} \mathrm{COO}\right)\right]^{-}
\end{aligned}
$$

We shall first examine the adsorption of $\mathrm{CH}_{3} \mathrm{COOH}$ and $\mathrm{CH}_{3} \mathrm{COO}^{-}$on the $I_{\mathrm{h}}$ isomer. The resulting optimized geometries are given in Figure 8, together with pMPA charges. $\left(\mathrm{CH}_{3} \mathrm{COO}\right)$ adsorbs on an edge, thus making a five-membered dimetallacycle with the metal surface. The hydrogen atom has been put opposite from $\left(\mathrm{CH}_{3} \mathrm{COO}\right)$ in order to avoid a close coupling of the electronic structure of surface species through shared metal atoms. Whereas a single $\mu_{3}-\mathrm{H}$ is more stable on $\mathrm{Ru}_{13}-I_{\mathrm{h}}$ than $\mu-\mathrm{H}$ by $3.3 \mathrm{kcal}^{\mathrm{mol}}{ }^{-1}, \mathrm{H}$ prefers an edge site in this system, with an energy difference of $\sim 2$ $\mathrm{kcal} . \mathrm{mol}^{-1}$ between the two isomers. The extra electron does not have a significant influence on geometries of the $\mathrm{CH}_{3} \mathrm{COO}$ moiety nor on the adsorption site, except for the $\mathrm{Ru}-\mathrm{O}$ bond lengths which are slightly elongated by $\sim 0.01 \AA$ in $\left[\left(\mathrm{Ru}_{13}-\mathrm{I}_{\mathrm{h}}\right)\left(\mathrm{CH}_{3} \mathrm{COO}\right)\right]^{-}$with respect to $\left(\mathrm{Ru}_{13}\right)\left(\mathrm{CH}_{3} \mathrm{COO}\right)(\mathrm{H})$. With a -0.38 e charge, the adsorbed hydrogen atom has clearly an hydridic character. $\mathrm{CH}_{3} \mathrm{COO}$ is also negatively charged in both clusters. Interestingly, whereas the $\mathrm{Ru}_{13}$ core is oxidized by the hydride and the ethanoate in the neutral cluster (the overall charge of $\mathrm{Ru}_{13}$ is $1.32 e$ ), a reduction of
$\mathrm{Ru}_{13}$ is observed in $\left[\left(\mathrm{Ru}_{13}\right)\left(\mathrm{CH}_{3} \mathrm{COO}\right)\right]^{-}$. By comparison with the neutral cluster, it means that the extra electron is mainly delocalized within the metal core. The two ruthenium atoms involved in the coordination of the ethanoate moiety in the anionic cluster possess however the same positive charge as in the neutral one. The pDOS and pCOHP profiles of the two clusters are shown in Figure S1. As already observed in the bare clusters, the Fermi energy, $E_{F}$, increases in the negatively charged cluster with respect to the neutral one. Apart from this shift, the pCOHP and pDOS profiles look similar at first sight. However, the $\mathrm{IpCOHP}(\mathrm{Ru}-$ $\mathrm{Ru}$ ) index is weaker by $2.3 \mathrm{kcal} . \mathrm{mol}^{-1}$ per Ru-Ru bond in the anionic cluster, in line with a delocalization of the extra charge mainly on the metal part and with the anti-bonding metal character of the electronic states around the Fermi energy.

The adsorption of one ethanoate ion on $\mathrm{Ru}_{13}-I_{\mathrm{h}}$ (reaction 4) is stronger than the dissociative adsorption of a single ethanoic acid molecule (reaction 3 ) by $\sim 20$ kcal.mol ${ }^{-1}\left(E_{\text {ads }}(\mathrm{RCOOH}):-56.6 \mathrm{kcal}^{\mathrm{mol}}{ }^{-1}\right.$ vs. $E_{\mathrm{ads}}\left(\mathrm{RCOO}^{-}\right)$: -76.9 kcal.mol ${ }^{-1}$ ). Provided that the carboxylate-metal bond strengths be quite similar in the anionic and neutral clusters, and despite the lowering of the metal cohesive energy in the anionic cluster, this significant energy difference is explained by a high $\mathrm{O}-\mathrm{H}$ bond dissociation energy that is not counterbalanced by the newly formed $\mathrm{Ru}-\mathrm{H}$ bond in the neutral cluster.

We shall now qualitatively examine the adsorption energies, $E_{\text {ads }}(\mathrm{RCOOH})$ and $E_{\text {ads }}\left(\mathrm{RCOO}^{-}\right)$as a function of acid strength. The dissociative and direct adsorption energies, $E_{\text {ads }}(\mathrm{RCOOH})$ and $E_{\text {ads }}\left(\mathrm{RCOO}^{-}\right)$, of seven carboxylic acids/conjugate bases at the surface of the $\mathrm{Ru}_{13}-I_{\mathrm{h}}$ and $\mathrm{Ru}_{13}-C_{\mathrm{s}}-1$ clusters are reported in Figure 9 as a function of experimental gas-phase acidities, $\Delta_{a} H^{\circ}$ (the hydride is found to be $\mu_{3}$ in the $C_{\mathrm{s}}-1$ isomer). Four main results arise: the linear relationships between experimental gas-phase acidities and adsorption energies of acids and bases at the surface of both clusters; the slight increase of adsorption strengths of acids and the strong decrease of adsorption strengths of bases as a function of the acid strength; the stronger adsorption of bases, with the exception of heptafluorobutanoic acid on the icosahedral isomer; very similar adsorption energies are found for the $C_{\mathrm{s}^{-}}-1$ and $I_{\mathrm{h}}$ isomers, despite different motif-based clusters.

\section{Conclusion}

Regarding acidities, this study shows that backgroundcorrected DFT-PBE with periodic boundary conditions performs very well. Enthalpies differ generally by less than $1 \mathrm{kcal} . \mathrm{mol}^{-1}$ with respect to non-periodic PBE 

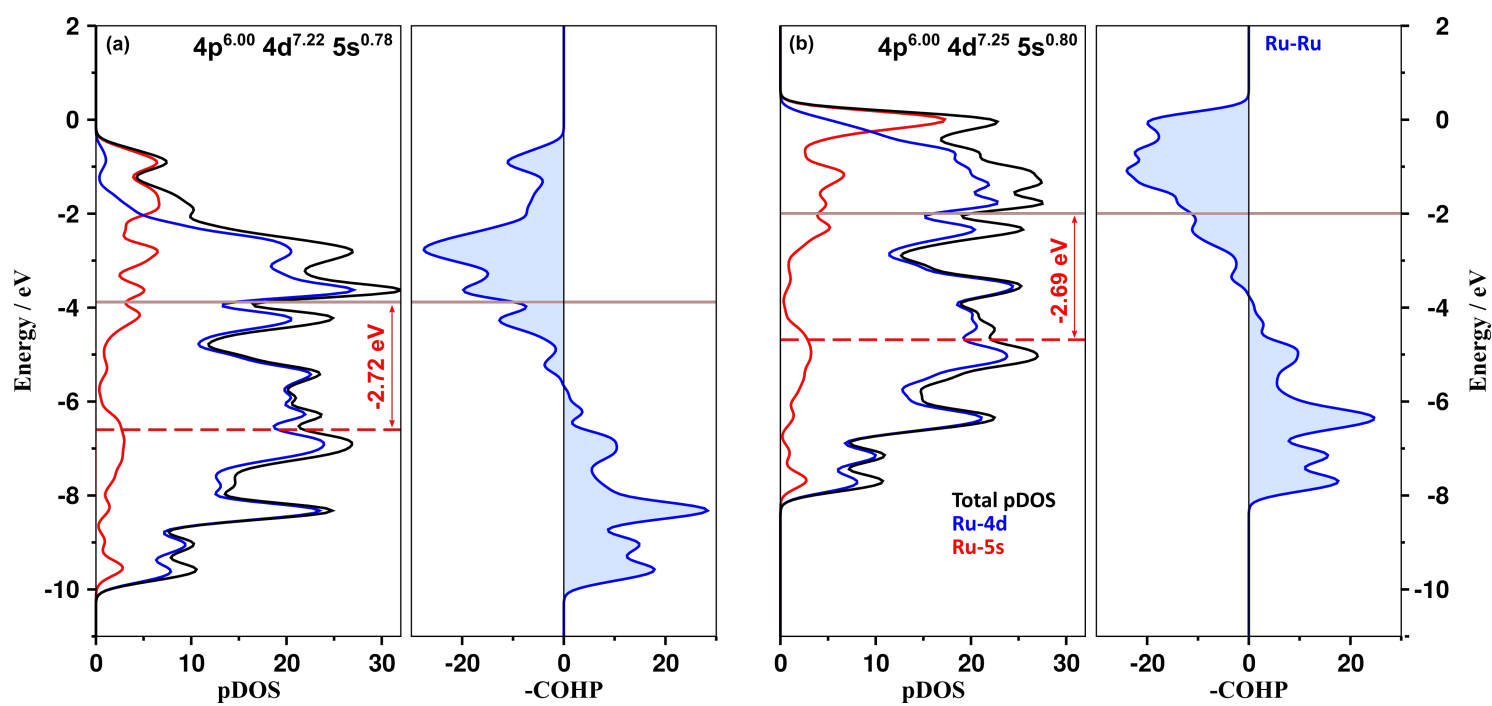

Fig. 7 Projected DOS and COHP analysis for the nearest neighbor interactions in the bare $\mathrm{Ru}_{13}^{\mathrm{q}}-\mathrm{C}_{2 \mathrm{v}}$ clusters. (a) $q=0$; (b) $q=-1$. The average occupation of the $4 p, 4 d$ and $5 s$ bands are also reported, as well as the $d$-band center for all Ru atoms (dashed red line) and the position of the Fermi level (brown horizontal line). The low-lying $4 p$-DOS does not appear in this energy range. pCOHP profiles are calculated for nearest neighbors only

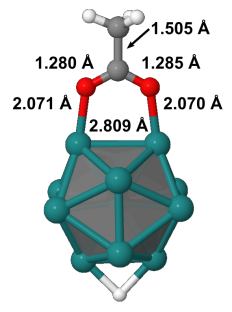

(a) $\left(\mathrm{Ru}_{13}-\mathrm{I}_{\mathrm{h}}\right)\left(\mathrm{CH}_{3} \mathrm{COO}\right)(\mathrm{H})$

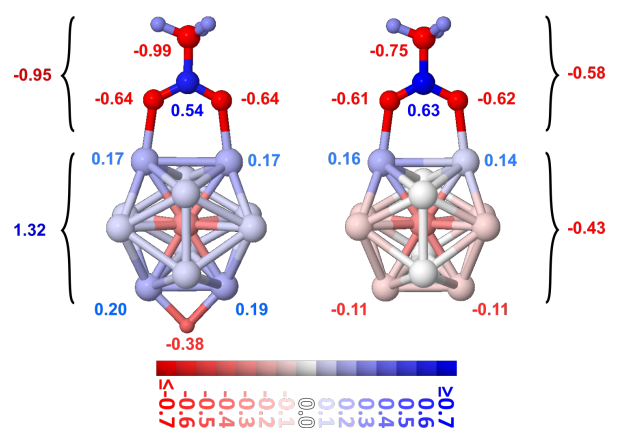

Fig. 8 Adsorption on the $\mathrm{Ru}_{13}-I_{\mathrm{h}}$ cluster of a dissociated ethanoic acid (a); of ethanoate (b). Atomic pMPA charges (in units of $e$ ), shown as color maps, are given below, together with the color reference charge scale.

calculations with polarized triple-zeta gaussian basis augmented with diffuse functions (aug-cc-pvtz). When compared to experimental reference enthalpies, the mean average deviation (MAD) is low (ca. $\left.4 \mathrm{kcal}_{\mathrm{mol}}{ }^{-1}\right)$. In addition to this satisfactory agreement between methods, this study also underlines that GGA functionals well describe proton-transfer reactions in the gas phase. The main failure was found for the hydroxide anion, and

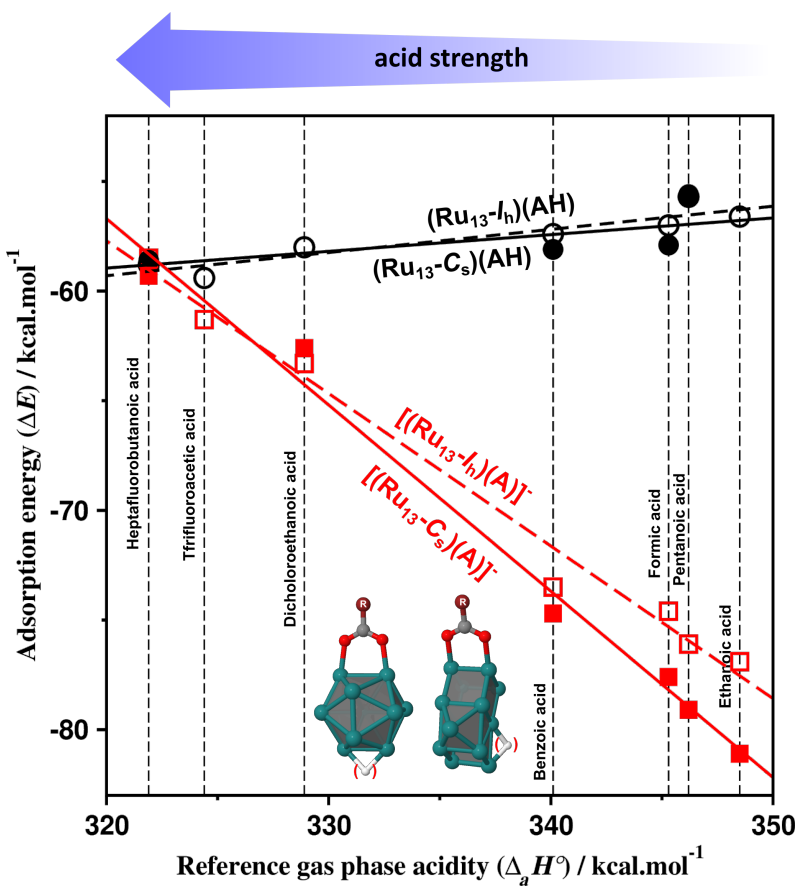

Fig. 9 Comparison of adsorption energies on the $\mathrm{Ru}_{13}-I_{\mathrm{h}}$ (dashed lines and empty symbols) and $\mathrm{Ru}_{13}-C_{\mathrm{S}^{-}}-1$ (plain lines and filled symbols) clusters calculated in this work with experimental gas-phase acidities $\Delta_{a} H^{\circ}$ (see references in the text and in table 1). Black circles and lines: dissociative adsorption energy of RCOOH acids, $E_{\text {ads }}(\mathrm{RCOOH})$; Red squares and lines: adsorption energy of $\mathrm{RCOO}^{-}$bases, $E_{\mathrm{ads}}\left(\mathrm{RCOO}^{-}\right)$. The adsorption sites are also shown.

especially for the methyl anion. The relative ordering of three $\mathrm{Ru}_{13}^{-}$isomers is close within a few kcal.mol ${ }^{-1}$ to TPSS results obtained using a local basis set. Finally, we use these good results to calculate the adsorp- 
tion properties of carboxylic acids and carboxylates on two $\mathrm{Ru}_{13}^{-}$isomers as a function of acid strength. Weak acids are less strongly bound to the metal surface than their conjugate bases. Dissociative adsorption energies of acids vary within a narrow energy range as a function of acid strength, whereas adsorption energies of bases are more sensitive to this property. An evaluation of these trends on ligand- and hydride-protected models is ongoing. More generally, such case study provides new perspectives in the theoretical treatment of both colloidal ultra-small TMNPs stabilized with charged species and of ionized TM clusters produced in mass spectrometry-based molecular physics experiments. It also offers an alternative to theoretically describe catalytic processes that involve charged species, such as redox reactions.

Acknowledgements We acknowledge HPCs CALcul en MIdiPyrénées (CALMIP-Olympe, grant P0611) and the Grand Equipement National de Calcul Intensif (GENCI-TGCC, grant 6211) for generous allocations of computer time. R. González-Gómez thanks the Consejo Nacional de Ciencia y Tecnología (CONACyT) for financing his $\mathrm{PhD}$ scholarship (grant CVU-483283). Université Paul Sabatier-Toulouse, INSAT and CNRS are also thanked for financial support. Special thanks goes to Dr. Detlef Schooss for his valuable comments.

\section{References}

1. Wilcoxon, J. P.; Abrams, B. L. Synthesis, structure and properties of metal nanoclusters. Chem. Soc. Rev. 2006, 35, 1162.

2. Burda, C.; Chen, X. B.; Narayanan, R.; El-Sayed, M. A. Chemistry and properties of nanocrystals of different shapes. Chem. Rev. 2005, 105, 1025-1102.

3. Schmid, G., Ed. Nanoparticles. From theory to application, 2nd ed.; Wiley-VCH: Weinheim, Germany, 2010.

4. Amiens, C.; Ciuculescu-Pradines, D.; Philippot, K. Controlled metal nanostructures: Fertile ground for coordination chemists. Coord. Chem. Rev. 2016, 308, 409-432.

5. Martínez-Prieto, L. M.; Chaudret, B. Organometallic Ruthenium Nanoparticles: Synthesis, Surface Chemistry, and Insights into Ligand Coordination. Acc. Chem. Res. 2018, 51, $376-384$.

6. Schooss, D.; Blom, M. N.; Parks, J. H.; v. Issendorff, B.; Haberland, H.; Kappes, M. M. The Structures of $\operatorname{Ag}_{55}^{+}$ and $\mathrm{Ag}_{55}^{-}$: Trapped Ion Electron Diffraction and Density Functional Theory. Nano Lett. 2005, 5, 1972-1977, PMID: 16218720 .

7. Lechtken, A.; Neiss, C.; Kappes, M. M.; Schooss, D. Structure determination of gold clusters by trapped ion electron diffraction: Au14--Au19-. Phys. Chem. Chem. Phys. 2009, 11, 4344 .

8. Rapps, T.; Ahlrichs, R.; Waldt, E.; Kappes, M. M.; Schooss, D. On the Structures of 55-Atom Transition-Metal Clusters and Their Relationship to the Crystalline Bulk. Angew. Chem. Int. Ed. 2013, 52, 6102-6105.

9. Waldt, E.; Ahlrichs, R.; Kappes, M. M.; Schooss, D. Structures of Medium-Sized Ruthenium Clusters: The Octahedral Motif. ChemPhysChem 2014, 15, 862-865.
10. Bumüller, D.; Hehn, A.-S.; Waldt, E.; Ahlrichs, R.; Kappes, M. M.; Schooss, D. Ruthenium Cluster Structure Change Induced by Hydrogen Adsorption: Ru19-. J. Phys. Chem. C 2016,

11. Kresse, G.; Fürthmuller, J. Efficient Iterative Schemes for $a b$ initio Total-Energy Calculations Using a Plane-Wave Basis Set. Phys. Rev. B 1996, 54, 11169-11186.

12. Kresse, G.; Fürthmuller, J. Efficiency of $a b$ initio Total Energy Calculations for Metals and Semiconductors Using a Plane-Wave Basis Set. Comput. Mater. Sci. 1996, 6, 15-50.

13. TURBOMOLE, a development of University of Karlsruhe and Forschungszentrum Karlsruhe GmbH, 1989-2007, TURBOMOLE GmbH, since 2007; available from http://www.turbomole.com.

14. Neugebauer, J.; Scheffler, M. Adsorbate-substrate and adsorbate-adsorbate interactions of $\mathrm{Na}$ and $\mathrm{K}$ adlayers on Al(111). Phys. Rev. B 1992, 46, 16067-16080.

15. Makov, G.; Payne, M. C. Periodic boundary conditions in $a b$ initio calculations. Phys. Rev. B 1995, 51, 4014-4022.

16. Frisch, M. J. et al. Gaussian 09 Revision D.01. Gaussian Inc. Wallingford CT 2009.

17. Perdew, J. P.; Burke, K.; Ernzerhof, M. Generalized Gradient Approximation Made Simple. Phys. Rev. Lett. 1996, 77, 3865-3868.

18. Perdew, J. P.; Burke, K.; Ernzerhof, M. Generalized Gradient Approximation Made Simple [Phys. Rev. Lett. 77, 3865 (1996)]. Phys. Rev. Lett. 1997, 78, 1396.

19. Dunning, T. H. Gaussian basis sets for use in correlated molecular calculations. I. The atoms boron through neon and hydrogen. J. Chem. Phys. 1989, 90, 1007-1023.

20. Kendall, R. A.; Dunning, T. H.; Harrison, R. J. Electron affinities of the first-row atoms revisited. Systematic basis sets and wave functions. J. Chem. Phys. 1992, 96, 67966806.

21. Woon, D. E.; Dunning, T. H. Gaussian-Basis Sets for Use in Correlation Molecular Calculations. III. The Atoms Aluminium Through Argon. J. Chem. Phys. 1993, 98, 13581371.

22. McQuarrie, D. A.; Simon, J. D. Molecular Thermodynamics; University Science Book: Sausalito, California, 1999.

23. Blöchl, P. Projector Augmented-Wave Method. Phys. Rev. B 1994, 50, 17953-17979.

24. Kresse, G.; Joubert, D. From Ultrasoft Pseudopotentials to the Projector Augmented-Wave Method. Phys. Rev. B 1999, 59, 1758-1775.

25. Monkhorst, H. J.; Pack, J. D. Special Points for BrillouinZone Integrations. Phys. Rev. B 1976, 13, 5188-5192.

26. Hammer, B.; Nørskov, J. K. Electronic Factors Determining the Reactivity of Metal Surfaces. Surf. Sci. 1995, 343, 211220 .

27. del Rosal, I.; Mercy, M.; Gerber, I. C.; Poteau, R. LigandField Theory-Based Analysis of the Adsorption Properties of Ruthenium Nanoparticles. ACS Nano 2013, 7, 9823-9835.

28. Dronskowski, R.; Blöchl, P. E. Crystal Orbital Hamilton Populations (COHP): Energy-Resolved Visualization of Chemical Bonding in Solids Based on Density-Functional Calculations. J. Phys. Chem. 1993, 97, 8617-8624.

29. Deringer, V. L.; Tchougréeff, A. L.; Dronskowski, R. Crystal Orbital Hamilton Population (COHP) Analysis As Projected from Plane-Wave Basis Sets. J. Phys. Chem. A 2011, 115, 5461-5466.

30. Maintz, S.; Deringer, V. L.; Tchougréeff, A. L.; Dronskowski, R. Analytic projection from plane-wave and PAW wavefunctions and application to chemical-bonding analysis in solids. J. Comp. Chem. 2013, 34, 2557-2567. 
31. Maintz, S.; Deringer, V. L.; Tchougréeff, A. L.; Dronskowski, R. LOBSTER: A tool to extract chemical bonding from plane-wave based DFT. J. Comp. Chem. 2016, 37, 1030-1035.

32. Cusinato, L.; Martinez-Prieto, L. M.; Chaudret, B.; del Rosal, I.; Poteau, R. Theoretical characterization of the surface composition of ruthenium nanoparticles in equilibrium with syngas. Nanoscale 2016, 8, 10974-10992.

33. Cusinato, L.; del Rosal, I.; Poteau, R. Shape, electronic structure and steric effects of organometallic nanocatalysts: relevant tools to improve the synergy between theory and experiments. Dalton Trans. 2017, 46, 378-395.

34. Gerber, I. C.; Poteau, R. Critical assessment of charge transfer estimates in non-covalent graphene doping. Theo. Chem. Acc. 2018, 137, 156.

35. Poad, B. L. J.; Reed, N. D.; Hansen, C. S.; Trevitt, A. J.; Blanksby, S. J.; Mackay, E. G.; Sherburn, M. S.; Chan, B.; Radom, L. Preparation of an ion with the highest calculated proton affinity: ortho-diethynylbenzene dianion. Chem. Sci. 2016, 7, 6245-6250.

36. Caldwell, G.; Renneboog, R.; Kebarle, P. Gas phase acidities of aliphatic carboxylic acids, based on measurements of proton transfer equilibria. Can. J. Chem. 1989, 67, 611-618.

37. Chase Jr., M. W.; Davies, C. A.; Downey Jr, J. R.; Frurip, D. J.; McDonald, R. A.; Syverud, A. N. JANAF Thermochemical Tables, 3rd ed.; U.S. National Bureau of Standards, 1985; Vol. 14.

38. Morris, R. A.; Knighton, W. B.; Viggiano, A. A.; Hoffman, B. C.; III, H. F. S. The gas-phase acidity of $\mathrm{H}_{3} \mathrm{PO}_{4} . J$. Chem. Phys. 1997, 106, 3545-3547.

39. Hunter, E. P. L.; Lias, S. G. Evaluated Gas Phase Basicities and Proton Affinities of Molecules: An Update. J. Phys. Chem. Ref. Data 1998, 27, 413-656.

40. Angel, L. A.; Ervin, K. M. Gas-Phase Acidities and O-H Bond Dissociation Enthalpies of Phenol, 3-Methylphenol, 2,4,6-Trimethylphenol, and Ethanoic Acid. J. Phys. Chem. A 2006, 110, 10392-10403.

41. Oliveira, A. M.; Lu, Y.-J.; Lehman, J. H.; Changala, P. B.; Baraban, J. H.; Stanton, J. F.; Lineberger, W. C. Photoelectron Spectroscopy of the Methide Anion: Electron Affinities of ${ }^{\bullet} \mathrm{CH}_{3}$ and $\bullet \mathrm{CD}_{3}$ and Inversion Splittings of $\mathrm{CH}_{3}^{-}$and $\mathrm{CD}_{3}^{-}$. J. Am. Chem. Soc. 2015, 137, 12939-12945.

42. Linstrom, P. J., Mallard, W. G., Eds. NIST Chemistry WebBook, NIST Standard Reference Database Number 69; National Institute of Standards and Technology: Gaithersburg MD, 20899, USA, 2005.

43. Waldt, E.; Hehn, A.-S.; Ahlrichs, R.; Kappes, M. M.; Schooss, D. Structural evolution of small ruthenium cluster anions. J. Chem. Phys. 2015, 142, 024319.

44. Tao, J.; Perdew, J. P.; Staroverov, V. N.; Scuseria, G. E. Climbing the Density Functional Ladder: Nonempirical Meta-Generalized Gradient Approximation Designed for Molecules and Solids. Phys. Rev. Lett. 2003, 91.

45. Weigend, F.; Ahlrichs, R. Balanced basis sets of split valence, triple zeta valence and quadruple zeta valence quality for $\mathrm{H}$ to Rn: Design and assessment of accuracy. Phys. Chem. Chem. Phys. 2005, 7, 3297.

46. Truflandier, L. A.; del Rosal, I.; Chaudret, B.; Poteau, R.; Gerber, I. C. Where does hydrogen adsorb on Ru nanoparticles ? A powerful joint ${ }^{2} \mathrm{H}$ MAS-NMR / DFT study. ChemPhysChem 2009, 10, 2939-2942.

47. Taglang, C.; Martínez-Prieto, L. M.; del Rosal, I.; Maron, L.; Poteau, R.; Philippot, K.; Chaudret, B.; Perato, S.; Sam Lone, A.; Puente, C.; Dugave, C.; Rousseau, B.; Pieters, G. Enantiospecific C-H Activation Using Ruthenium Nanocatalysts. Angew. Chem., Int. ed. Eng. 2015, 54,
10474-10477.

48. Martínez-Prieto, L. M.; Cano, I.; Márquez, A.; Baquero, E. A.; Tricard, S.; Cusinato, L.; del Rosal, I.; Poteau, R.; Coppel, Y.; Philippot, K.; Chaudret, B.; Cámpora, J.; ; van Leeuwen, P. W. N. M. Zwitterionic amidinates as effective ligands for platinum nanoparticle hydrogenation catalysts. Chem. Sci. 2017, 8, 2931-2941.

49. Herron, J. A.; Tonelli, S.; Mavrikakis, M. Atomic and molecular adsorption on $\mathrm{Ru}(0001)$. Surf. Sci. 2013, 614, 64-74. 